\title{
Modelo matemático de transporte para una empresa comercializadora de combustibles, usando programación lineal
}

\section{Mathematical model of transport for a company commercializing fuels, using linear programming}

Jenny Margoth Villamarín Padilla. ${ }^{1}$, Gustavo Javier Aguilar Miranda. ${ }^{2}$, José Luis Llamuca Llamuca. ${ }^{3}$ \& Wilfrido Humberto Villacrés Suárez. ${ }^{4}$

\section{DOI: https://doi.org/10.33262/visionariodigital.v3i2.394}

\section{Resumen.}

El objetivo de este artículo es determinar un modelo matemático de transporte usando programación lineal. Para resolver este problema, según la metodología del algoritmo de transporte, se construye una tabla o matriz de distribución colocando en cada renglón a los centros de suministro y en cada columna a los centros de consumo, para llevar a cabo esta distribución se recolecta información de la demanda, se definió la flota de vehículos, el tiempo de recorrido, las tarifas y los costos de realizar cada viaje, para luego planear nuestro modelo de programación lineal con la utilización del software Microsoft Excel, con esta información se realiza el estudio de los diferentes métodos de transporte eficiente aplicables a las operaciones de la compañía, con sus principales características, ventajas y desventajas, teniendo en cuenta la problemática de la planeación y programación asociados, con los resultados obtenidos por los cinco métodos estudiados, se puede concluir que el modelo matemático de transporte óptimo para la empresa comercializadora de combustibles, basado en programación lineal, es el que presenta la red generada por el

\footnotetext{
${ }^{1}$ Escuela Superior Politécnica de Chimborazo, Facultad de Administración de Empresas, Riobamba, Ecuador, j_villamarin@espoch.edu.ec

2 Escuela Superior Politécnica de Chimborazo, Facultad de Administración de Empresas, Riobamba, Ecuador, gustavo.aguilar@espoch.edu.ec

${ }^{3}$ Escuela Superior Politécnica de Chimborazo, Facultad de Administración de Empresas, Riobamba, Ecuador, jose.llamuca@espoch.edu.ec

${ }^{4}$ Escuela Superior Politécnica de Chimborazo, Facultad de Administración de Empresas, Riobamba, Ecuador, wvillacrés@espoch.edu.ec
} 
método del costo menor, Vogel y Russel, que proponen la misma estructura de despachos y abastecimientos, y generan un costo mínimo de \$206354,00 dólares.

Palabras claves: modelo matemático, transporte, programación, comercializadora, lineal.

\section{Abstract.}

The objective of this article is to determine a mathematical model of transport using linear programming. To solve this problem, according to the methodology of the transport algorithm, a distribution table or matrix is constructed by placing the supply centers in each line and in each column the consumption centers, in order to carry out this distribution information of the demand, the fleet of vehicles was defined, the time of travel, the fares and the costs of making each trip, to then plan our linear programming model with the use of Microsoft Excel software, with this information the study of the different methods of efficient transport applicable to the company's operations, with their main characteristics, advantages and disadvantages, taking into account the associated planning and programming problems, with the results obtained by the five methods studied, it can be concluded that the model optimum transport mathematician for the fuel trading company, based on linear programming, is the one that presents the network generated by the lowest cost method, Vogel and Russel, which propose the same structure of dispatches and supplies, and generate a minimum cost of \$206354.00 dollars.

Keywords: mathematical model, transport, programming, marketer, linear

\section{Introducción.}

La programación de la distribución se encarga de optimizar la asignación de las tareas a realizar (entrega de pedidos) con los recursos disponibles (vehículos). Específicamente las industrias tienen como objetivo optimizar el flujo de productos desde los centros de producción hasta el usuario (cliente).

Medina, Raya \& Contreras (2007) describen un modelo de programación lineal diseñado en Excel para asignar trabajos a máquinas considerando prioridades específicas por planeadores de una empresa manufacturera, el mismo que incrementa significativamente la eficiencia de la operación actual. Así mismo, López, Olguín \& Camargo (2008) presentan un modelo de transporte usando programación lineal. (Adame, Fontanilla, \& Arango, 2011) proponen dos modelos logísticos aplicables en la gestión del transporte en la agroindustria de la palma de aceite en Colombia, de igual manera García (2014) realiza un estudio cualitativo de los diferentes modelos de transporte eficiente aplicables a las operaciones de las compañías del sector de hidrocarburos en Colombia, teniendo en cuenta la problemática de la planeación y programación asociados. 
Las restricciones de los estudios anteriores se hacen más evidentes en el caso de empresas que por sus características requieren una programación en el corto plazo. En los últimos años, la mayor parte de los estudios desarrollados en el ámbito de los modelos de transporte mediante la programación lineal se concentra en el diseño de la estructura general de la distribución, ampliándolo en algunos casos a otros procesos logísticos de la empresa.

En este artículo se presenta la optimización de la distribución, mediante programación matemática para empresas que necesitan una programación a corto plazo y que tiene las siguientes características: Flota de vehículos disponibles conocida, distribución basada en transporte directo origen - destino, distribución entre varios orígenes y diferentes destinos.

Esta caracterización considera algunos aspectos como la distancia entre los diferentes orígenes y destinos, sobre todo en el transporte entre los terminales de almacenaje y las estaciones de servicio, ya que se pretende reducir significativamente los costos de transporte.

Para ello se ha utiliza cinco métodos de transporte diferentes para establecer el modelo óptimo, es decir el modelo que genere el menor costo, como presenta (Adame, Fontanilla, \& Arango, 2011), al igual que García (2014) analiza los modelos de transporte eficiente aplicables a las operaciones de las compañías.

El modelo que se presenta minimiza el costo de transporte para la empresa de comercializadora de combustibles en estudio, considerando cuatro terminales de almacenamiento (Santo Domingo, Quito, Ambato y Riobamba-San Juan ) y cuatro estaciones de servicio (Tulcán, Quito, Ambato, Riobamba), considerando una flota de vehículos fija, se estudian cinco posibilidades distintas según los métodos de transporte escogidos: método de la esquina noroeste, costo menor, mutuamente preferente, Voguel y Russel.

\section{Desarrollo.}

El modelo de transporte involucra el embarque de ciertos artículos o productos homogéneos desde diferentes orígenes hacia varios destinos (Puente \& Gavilánez, 2018).

Cada uno de los orígenes representa la fuente de suministro del artículo o producto, cada destino representa el punto de demanda. El modelo de distribución (modelo de transporte) es un conjunto importante del problema de optimización de redes, ha sido aplicado al control y diseño de plantas de fabricación, determinación de territorios de venta, localización de centros de distribución y almacenaje. A este método suele nombrarse como de transporte por la similitud existente en el planteamiento de problemas de fletar mercaderías desde los sitios de producción hasta los lugares de consumo (Izar, 2012).

La red que aparece en la figura 1 representa el problema. Hay $m$ orígenes y $n$ destinos, cada uno representado por un nodo. Los arcos representan las rutas que unen los orígenes con los destinos. El arco $(i, j)$ que une el origen $i$ con el destino $j$ transporta dos piezas de 
información: el costo de transporte por unidad, $\mathrm{c}_{i j} \mathrm{y}$ la cantidad transportada, $\mathrm{x}_{i j}$. La cantidad de la oferta en el origen $i$ es $a_{i}$ y la cantidad de la demanda en el destino $j$ es $b_{j}$. El objetivo del modelo es minimizar el costo de transporte total al mismo tiempo que se satisfacen las restricciones de la oferta y la demanda (Taha, 2012).

Figura 1. Representación del modelo de transporte con nodos y arcos.

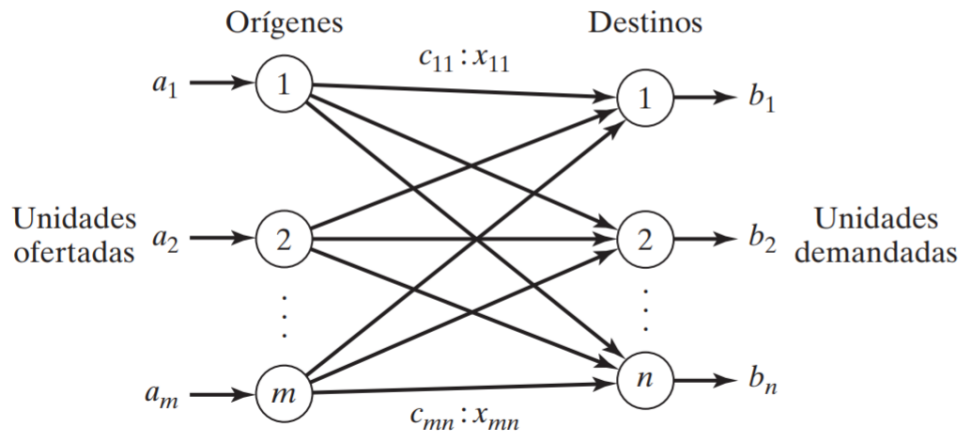

Fuente: (Taha, 2012).

\section{Modelos de transporte.}

Taha (2012) en su obra Investigación de operaciones dice lo siguiente:

Un modelo de transporte general con $m$ orígenes y $n$ destinos tiene $m+n$ ecuaciones de restricción, una por cada origen y cada destino. Sin embargo, como el modelo de transporte siempre está balanceado (suma de la oferta = suma de la demanda) una de las ecuaciones es redundante, por lo que el modelo se reduce a $\mathrm{m}+\mathrm{n}-1$ ecuaciones independientes y $\mathrm{m}+\mathrm{n}$ 1 variables básica. La estructura especial del problema de transporte permite asegurar una solución básica no artificial siguiendo uno de los métodos de transporte (pág. 188).

Puente y Gavilánez (2018) en su obra Programación lineal para la toma de decisiones manifiesta lo siguiente:

Existen diferentes métodos de transporte entre los cuales se puede destacar: (pág. 62)

Métodos de inicialización.

- Método de la esquina noroeste

- Método del costo menor

- Método mutuamente preferente

- Método Vogel

- Método Russel 
Métodos de Optimización.

- Método del cruce del arroyo

- Método MODI

\section{Método de la esquina noroeste.}

Este método de la esquina noroeste es el más sencillo para lograr la distribución inicial, es el menos recomendado, pues el costo de la matriz inicial es muy elevado. Los pasos para desarrollar este método son los siguientes:

1. Se inicia la distribución por la casilla de la esquina noroeste de la tabla, asignándole el máximo que sea posible. Se selecciona el número menor entre oferta y demanda que corresponde a la casilla, para satisfacer la oferta o la demanda. Al renglón o columna que haya sido satisfecho, se le asignarán ceros, para que la sumatoria de la oferta o la demanda no se altere, creándose así una tabla de menor tamaño.

2. En la matriz que no tiene asignación en el paso anterior se localiza la nueva casilla noroeste y se repite el paso anterior.

3. Se repite todo el procedimiento anterior hasta que toda la tabla tenga una asignación. (pág. 63)

Este método de esquina noroeste corresponde a un método matricial del método simplex. Para determinar cuántos envíos se realizarán desde cada origen hacia cada destino se aplica la siguiente fórmula:

Número de envíos $=m+n-1$

Donde:

m: constituye el número de filas

n: el número de columnas

\section{Método del costo menor}

Este método de inicialización es más eficiente que el método de la esquina noroeste, pues va asignado el menor costo de entre las casillas, de ahí su nombre. Cabe mencionar que este método no siempre logra tener la solución óptima.

Su procedimiento se detalla a continuación:

1. Analizar la primera columna y, sobre ella, localizar la casilla que tenga el menor costo, al cual se asignara el máximo posible de tal manera que se satisfaga la oferta o demanda. En el caso de que la primera columna no quedará satisfecha con la 
asignación, se procede de la misma manera hasta que la columna quede totalmente satisfecha.

2. Se continúa con la segunda columna repitiendo el procedimiento anterior.

3. Se procede con el resto de las columnas hasta la última de la tabla, con lo cual la distribución inicial quedará totalmente satisfecha. (pág. 66)

\section{Método mutuamente preferente.}

Selecciona las casillas de menor costo bajo el criterio de que sean a la vez las más bajas del renglón y la columna a la que pertenece.

1. Identificar las casillas que tengan el costo mínimo, tanto en el renglón, como en la columna a la que pertenece.

2. Asignar a las casillas la cantidad máxima posible para satisfacer sea la oferta o la demanda.

3. El resto de la tabla se va asignando, repitiendo los pasos anteriores. (pág. 70)

\section{Método Vogel.}

Este método es el más utilizado para lograr una asignación Inicial en los problemas de transporte, pues no es complicado y da muy buenas aproximaciones iniciales, las cuales muchas veces son las distribuciones óptimas, de aquí el por qué es tan popular.

Consiste en los siguientes pasos:

1. Para cada renglón y cada columna, encontrar la diferencia de costo entre la casilla más barata y la que le sigue en costo.

2. En aquel renglón o columna dónde bicha diferencia sea la máxima, asignar lo máximo posible en la casilla de menor costo. En caso de empates en las diferencias buscadas, se selecciona al azar una de ellas.

3. Se elimina aquella línea (renglón o columna) qué haya quedado satisfecha con la asignación del pasó anterior.

4. Repetir los pasos anteriores Hasta completar la tabla de transporte.

\section{Método de Russel.}

Este método es comparable al Vogel en cuanto a la aproximación, respecto a la solución óptima que ambos tienen, solo que este método es menos popular que la anterior debido a que requiere de una mayor cantidad de trabajo. 
Consiste en calcular antes de cada asignación la cantidad $\Delta_{\mathrm{ij}}$ para cada casilla libre disponible, conforme a la siguiente ecuación:

$$
\Delta_{i j}=\propto_{i}+\beta_{j}-C_{i j}
$$

Dónde:

$\Delta_{\mathrm{ij}}$ : Coeficiente de la Castilla del renglón $i$, columna $j$

$\alpha_{\mathrm{i}}$ : Costo mayor de las castillas del renglón $i$

$\beta_{\mathrm{j}}$ : costo mayor de las castillas de la columna $j$

$\mathrm{C}_{\mathrm{ij}}$ : costo de la casilla del renglón $i$, columna $j$

De aquí se ira asignando aquella casilla que tenga el valor más elevado de $\Delta_{\mathrm{ij}}$.

El procedimiento por pasos es el siguiente:

1. Se calcula $\Delta_{\mathrm{ij}}$ para el total de las casillas vacías de la tabla de transporte

2. En la casilla que haya tenido el mayor valor de $\Delta_{\mathrm{ij}}$, hacer la máxima asignación posible. Esto agotará la oferta del renglón y/o la demanda de la columna. en el caso de haber varias Casillas empatadas con el máximo valor de ahí $\Delta_{\mathrm{ij}}$, se selecciona arbitrariamente una de ellas.

3. Se elimina la tabla aquella línea que haya quedado satisfecha en el pasó anterior.

4. Repetir el procedimiento desde el pasó uno al tres con las casillas que aún están vacías hasta terminar las asignaciones de la tabla completa.

\section{Método del cruce del arroyo.}

El método del cruce del arroyo, también denominado algoritmo de Stepping Stone (Izar, 2012), es un método de programación lineal que consiste en calcular cuál sería la variación del costo del envío de una unidad de cierto producto por cada una de las rutas posibles, es decir asignar cierta cantidad de artículos desde varios orígenes (fábricas) a un conjunto de destinos (clientes) de tal manera que se disminuyan los costos, hasta optimizar la función objetivo. Dada una distribución inicial que cumpla la condición de no degeneración, se podrá optimizar el problema de transporte evaluando cada casilla vacía del recorrido cerrado correspondiente, el cual consiste en asignar unidades a las casillas vacías, trasladándolas de una casilla determinada que sea de la misma columna o renglón, de manera que sigan cumpliéndose las igualdades de la suma de asignaciones de las casillas por renglón de la oferta del mismo y la suma de asignaciones de las casillas por columna de la demanda. La distribución será óptima cuando los recorridos cerrados para el total de casillas vacías resulten mayores o iguales a cero, es decir; ninguna resulte negativa. (pág. 74) 


\section{Método MODI.}

MODI, conocido como el método de los costos ficticios, consiste en añadir a la matriz de costos una fila y una columna que recogen costos ficticios determinados arbitrariamente (los números MODI), tal que permita calcular los índices de mejora para las celdas (casillas) no utilizadas. Se aplica la siguiente fórmula: (Puente \& Gavilánez, 2018)

$$
r_{1}+C_{i j}+k_{j}=0
$$

Dónde:

$\mathrm{r}_{1}$ : coeficiente $\mathrm{r}$ para el renglón $\mathrm{i}$

$\mathrm{C}_{\mathrm{ij}}$ : costo de la casilla asignada ubicada en el renglón i y la columna $\mathrm{j}$

$\mathrm{K}_{\mathrm{j}}$ : coeficiente $\mathrm{k}$ para la columna $\mathrm{j}$

\section{Metodologia.}

El modelo de programación lineal se implementa en el paquete computacional Microsoft Excel, en un ordenador Asus con procesador Core 17 de 2,4 GHz, con 8 Gb de RAM. La experimentación se basa en observar los diferentes resultados obtenidos de forma aleatoria a partir de los datos proporcionados por la empresa comercializadora de combustibles, comentada anteriormente.

La empresa sobre la cual se genera la adquisición de los datos se dedica a la comercialización de gasolina y diésel en todo el país, sin embargo, el estudio se enfoca en la zona centro y norte del país, específicamente en las ciudades de Tulcán, Quito, Ambato y Riobamba, que son las ciudades que acogen el mayor número de estaciones de servicio, y por lo tanto la mayor cantidad de ventas.

A cada una de estas estaciones la empresa sirve pedidos, de dos a cuatro veces por semana. Las distancias entre los clientes de las diferentes zonas, en comparación con las distancias a los centros de origen y la frecuencia de entregas semanal, permite considerar los diferentes modelos matemáticos de transporte, que permitan establecer cuál es el más apropiado para esta empresa.

Además, las estaciones de Servicio (clientes) permiten un margen de un día para la entrega del producto solicitado. Por lo general, el tamaño de cada pedido de los distintos clientes supone entre el setenta y cinco y el cien por ciento de la capacidad de carga de los transportes que es de 4000 y 6000 galones. A cada una de las estaciones se envía entre dos y cuatro tanqueros por semana. 
El modelo matemático de transporte y resolución de la programación de la distribución, cosiste en asignar o distribuir diferentes cantidades de objetos desde unos orígenes hacia unos destinos, buscando hacerlo de una manera óptima, es decir a un costo mínimo (o bien con una utilidad máxima). Los orígenes serán los centros de suministro u ofertas, mientras que los destinos serán los centros de consumo o demandantes (Izar, 2012)

El estudio se realiza con cinco modelos de transporte, el que genere el menor costo será el óptimo y permitirá definir el modelo matemático de transporte para esta empresa.

\section{Planteamiento del problema de transporte}

Martínez, Vértiz, López, Jiménez, y Moncayo (2014) en su obra Investigación de operaciones manifiesta lo siguiente:

En el problema de transporte se busca la forma en que cualquier bien debe ser distribuido, desde cualquier grupo de centros de suministro (orígenes) a cualquier grupo de centros de recepción (destinos), de manera que los costos totales de transporte sean mínimos. (pág. 22)

Para que un caso pueda ser considerado problema de transporte, se debe cumplir con el supuesto de requerimientos y con el supuesto de costo.

El supuesto de requerimientos nos dice que cada origen tiene un suministro fijo de unidades y en suministro completo debe transportarse a los destinos. De manera análoga, cada destino tiene una demanda fija de unidades y debe satisfacerse de los orígenes. Es decir:

$$
\sum_{i=1}^{m} s_{i}=\sum_{j=1}^{n} d_{j} \quad \text { Ec. }(1)
$$

Dónde:

m: cantidad de orígenes

$\mathrm{n}$ : cantidad de destinos

$\mathrm{s}$ : cantidad de unidades que oferta el origen $\mathrm{i}$, para $i=1,2, \ldots, \mathrm{m}$

$\mathrm{d}$ : cantidad de unidades demandadas por el destino $j$, para $\mathrm{j}=1,2, \ldots, \mathrm{n}$

Dado que se debe priorizar la distribución de producto entre unidades reales y teniendo en mente que se busca minimizar, el supuesto de costo considera que el costo de transportar de un origen a un destino debe ser directamente proporcional al número de unidades transportadas. Este costo puede ser visto como el costo unitario multiplicado por el número de unidades transportadas. (Martínez, Vértiz, López, Jiménez, \& Moncayo, 2014)

Izar (2012) en su obra Investigación de operaciones manifiesta lo siguiente: 
Para plantear un problema típico de transporte tomaremos como ejemplo el caso de abastecer una mercancía desde 4 diferentes centros de suministro, A, B, C y E hacia 4 centros de consumo, W, U, Y, y Z, Buscando hacerlo a un costo total mínimo. (pág. 123)

Por su parte se tendrá que la oferta total P deberá ser igual a la demanda D.

La figura 2. nos muestra un diagrama del problema en donde vemos que habrá 4x4=16 diferentes caminos para transportar las mercancías desde los centros de suministro hasta los centros de consumo.

Figura 2. Diagrama del problema de los centros de suministro A, B, C, y E y de los centros de consumo W, U, Y, Z.

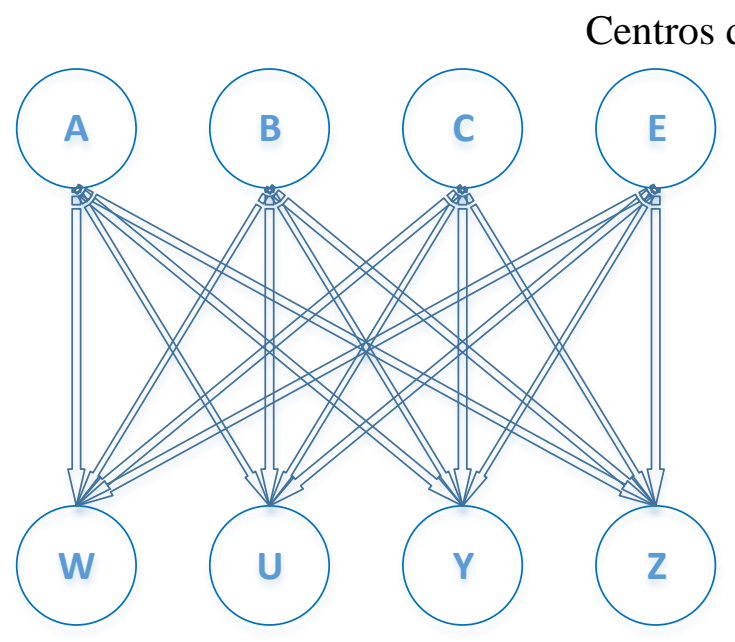

Fuente: (Izar, 2012)

\section{Centros de consumo}

Cada costo de envío de un centro de suministro a un centro de consumo se representa por Cij donde el subíndice $i$ representa el punto de suministro y j el de consumo. (pág. 124)

Si se plantea este problema como un caso de programación lineal, la función objetivo Z sería el costo total de los envíos, tal y como lo define la ecuación 2.

$$
\text { Minimizar } Z=\sum_{i=1}^{m} \sum_{j=1}^{n} C_{i j} X_{i j} \quad \text { Ec. (2) }
$$

Donde:

m: cantidad de orígenes

n: cantidad de destinos 
$\mathrm{c}_{\mathrm{ij}}$ : costo por transportar una unidad de producto desde el origen $i$ hasta el destino $j$ para $i=$ $1,2, \ldots \mathrm{m} ; j=1,2, \ldots \mathrm{n}$

$\mathrm{x}_{i j}$ : cantidad de unidades a enviar del orígen $i$ al destino $j$, para $i=1,2, \ldots \mathrm{m} ; j=1,2, \ldots \mathrm{n}$

La cual debería minimizarse.

Para resolver este problema, según la metodología del algoritmo de transporte, se construye una tabla o matriz de distribución colocando en cada renglón a los centros de suministro y en cada columna a los centros de consumo. Al final de cada renglón habrá una casilla para el total de las ofertas de los centros de suministro y al final de cada columna habrá una casilla para el total de las demandas de los centros de consumo.

En la Tabla 1, se muestra la matriz de distribución donde cada casilla estará disponible para el número de unidades que se enviarán del centro. de suministro que corresponde a ese renglón hacia el centro de consumo de esa columna.

Tabla 1: Matriz de transporte para el problema planteado de 4 centros de suministro y 4 centros de consumo.

\begin{tabular}{|c|c|c|c|c|c|}
\hline $\mathrm{O} \quad \mathrm{D}$ & $\mathrm{W}$ & $\mathrm{U}$ & $\mathrm{Y}$ & $\mathrm{Z}$ & OFERTAS \\
\hline$A$ & $\mathrm{C}_{\mathrm{AW}}$ & $\mathrm{C}_{\mathrm{AU}}$ & $\mathrm{C}_{\mathrm{AY}}$ & $\mathrm{C}_{\mathrm{AZ}}$ & $\mathrm{P}_{\mathrm{A}}$ \\
\hline $\mathrm{P}$ & $\mathrm{C}_{\mathrm{BW}}$ & $\mathrm{C}_{\mathrm{BU}}$ & $\mathrm{C}_{\mathrm{BY}}$ & $\mathrm{C}_{\mathrm{BZ}}$ & $\mathrm{P}_{\mathrm{B}}$ \\
\hline$C$ & $\mathrm{C}_{\mathrm{CW}}$ & $\mathrm{C}_{\mathrm{CU}}$ & $\mathrm{C}_{\mathrm{CY}}$ & $\mathrm{C}_{\mathrm{CZ}}$ & $\mathrm{P}_{\mathrm{C}}$ \\
\hline $\mathrm{E}$ & $\mathrm{C}_{\mathrm{EW}}$ & $\mathrm{C}_{\mathrm{EU}}$ & $\mathrm{C}_{\mathrm{EY}}$ & $\mathrm{C}_{\mathrm{EZ}}$ & $\mathrm{P}_{\mathrm{E}}$ \\
\hline DEMANDAS & $\mathrm{d}_{\mathrm{w}}$ & $\mathrm{d}_{\mathrm{u}}$ & $\overline{d_{y}}$ & $\mathrm{~d}_{\mathrm{z}}$ & $\mathrm{d}$ \\
\hline
\end{tabular}

Fuente: (Izar, 2012)

El recuadro superior derecho que aparece en cada casilla es para anotar en el al costo de enviar una unidad del centro de suministro del renglón en el que está ubicada la casilla hacia el centro de consumo de la columna correspondiente a la casilla. Así por ejemplo coma en la casilla $\mathrm{CZ}$ coma se llenará con el número de unidades que se enviaran desde $\mathrm{C}$ hasta $\mathrm{Z}$ siendo $\mathrm{C}_{\mathrm{CZ}}$ el costo unitario del envío.

Aquí el problema será como llenar la matriz de distribución de tal forma que se satisfagan las ofertas y demandas de cada renglón y columna respectivamente, logrando esto a un costo total que sea mínimo, es decir la suma de las asignaciones de cada renglón deberá ser 
igual a la oferta para ese renglón y la suma de las asignaciones de cada columna deberá ser la demanda de dicha columna coma no pudiendo haber ninguna asignación negativa, (Izar, 2012) es decir:

$X_{i j} \geq 0$

$$
\begin{aligned}
& \text { Para } i=1,2, \ldots, \mathrm{m} \\
& \text { Para } j=1,2, \ldots, \mathrm{m}
\end{aligned}
$$

\section{Resultados.}

Con la información obtenida de la empresa se ha construido las matrices de distribución (Ver Tabla 2) que permitirán la aplicación de los diferentes modelos de transporte, en este sentido la oferta lo constituyen el número de viajes que se requiere para el abastecimiento a las estaciones de servicio en las ciudades de Tulcán, Quito, Latacunga y Riobamba; la demanda está dada por los viajes de abastecimiento que pueden satisfacer los terminales o beaterios de las ciudades de Quito (Terminal 1), Santo Domingo (Terminal 2), Ambato (Terminal 3) y Riobamba (Terminal 4), de los cuales se transporta el combustible a las diferentes estaciones de servicio, de igual manera en la Tabla 1 se puede observar en cada una de las casillas que conforman la matriz, en la parte superior derecha, los costos de transporte asociados a cada una de las posibles rutas que se forman en la matriz, entre los terminales (orígenes) y las diferentes ciudades en las que existe estaciones de servicio de la comercializadora en estudio (destinos).

\begin{tabular}{|c|c|c|c|c|c|}
\hline $\mathrm{D}$ & Tulcán & Quito & Latacunga & Riobamba & Oferta \\
\hline \multirow{2}{*}{ Terminal 1} & 484 & 51 & 142 & 334 & \multirow[b]{2}{*}{420} \\
\hline & & & & & \\
\hline \multirow{2}{*}{ Terminal 2} & 774 & 223 & 312 & 502 & \multirow[b]{2}{*}{240} \\
\hline & & & & & \\
\hline \multirow{2}{*}{ Terminal 3} & 879 & 334 & 213 & 40 & \multirow[b]{2}{*}{150} \\
\hline & & & & & \\
\hline \multirow{2}{*}{ Terminal 4} & 772 & 225 & 83 & 109 & \multirow[b]{2}{*}{360} \\
\hline & & & & & \\
\hline Demanda & 210 & 450 & 180 & 330 & 1170 \\
\hline
\end{tabular}

Tabla 2. Matriz de distribución de transporte.

Fuente: Elaboración propia

La aplicación de cada uno de los modelos de transporte a la empresa en estudio se ha realizado considerando la planificación para cada destino de forma independiente y con una flotilla suficiente para que puedan servirse todos los pedidos. 
A continuación, se presentan los resultados obtenidos para cada uno de los modelos planteados, el modelo óptimo será el que minimice los costos del transporte.

\section{Análisis del método de transporte de la esquina noroeste.}

En la Tabla 3 se muestra los resultados del método de transporte de la Esquina noroeste, en la que se observa la satisfacción de todos los requerimientos establecidos por los destinos, en este caso a Tulcán se deberán enviar 210 tanqueros del terminal 1, a Quito se enviarán 210 tanqueros del terminal 1 y 240 tanqueros del terminal 2, a Latacunga se deben enviar 150 vehículos del terminal 3 y 30 del terminal 4, y por último a Riobamba, se enviarán 210 tanqueros del terminal 4, Siendo el objetivo final de los modelos la minimización de los costos, el plan determinado por las rutas escogidas tiene un costo de $\$ 223074,00$ dólares

Tabla 3. Matriz de distribución de transporte Modelo de la esquina noroeste

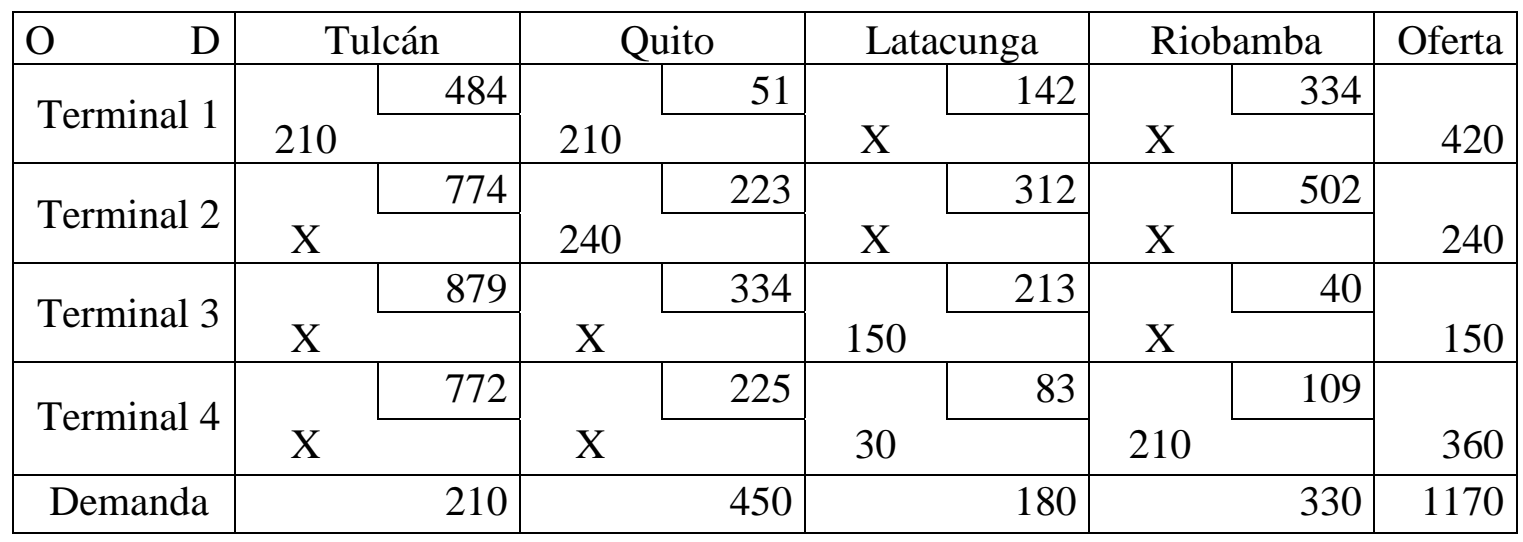

Fuente: Elaboración propia

$$
\begin{gathered}
C T=210(484)+210(51)+240(223)+150(213)+30(83)+210(109) \\
C T=223.074,00
\end{gathered}
$$

\section{Análisis método del costo menor}

En la Tabla 4 se muestra los resultados del método de transporte del costo menor, en el que se observa la siguiente estructura: a Tulcán se deberán enviar 210 tanqueros del terminal 1, a Quito se enviarán 210 tanqueros del terminal 1 y 240 tanqueros del terminal 2, a Latacunga se deben enviar 180 vehículos del terminal 4, y a Riobamba, se enviarán 150 tanqueros del terminal 3, y 180 del terminal 4. El plan determinado por las rutas escogidas por este método tiene un costo de $\$ 206354,00$ dólares. 
Tabla 4. Matriz de distribución de transporte Modelo del costo menor.

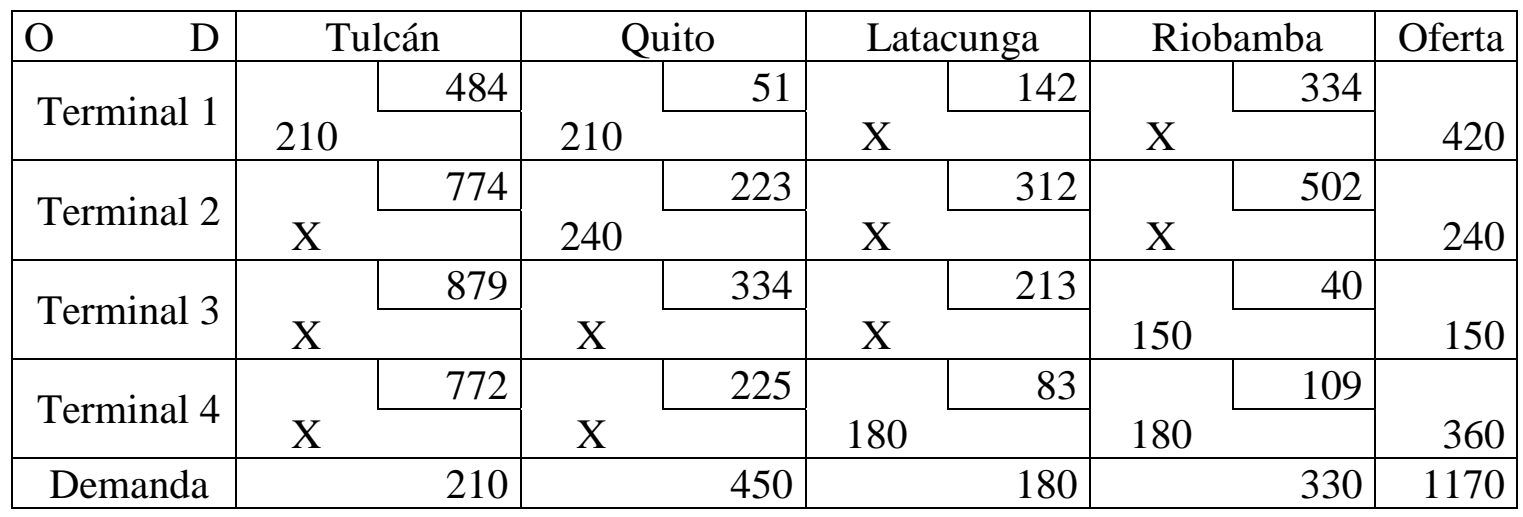

Fuente: Elaboración propia.

$$
\begin{gathered}
C T=210(484)+210(51)+240(223)+150(40)+180(83)+180(109) \\
C T=206.354,00
\end{gathered}
$$

\section{Análisis método mutuamente preferente}

En la Tabla 5, se muestra los resultados del método de transporte mutuamente preferente, en el que se observa la siguiente red: A Tulcán se deberán proveer 210 tanqueros del terminal 2, a Quito se enviarán 420 tanqueros del terminal 1 y 30 tanqueros del terminal 2, a Latacunga se deben enviar 180 vehículos del terminal 4, y a Riobamba, se enviarán 150 tanqueros del terminal 3, y 180 del terminal 4, Siendo el objetivo final del modelo la minimización de los costos, el plan determinado por las rutas escogidas tiene un costo de \$231091,00 dólares.

Tabla 5. Matriz de distribución de transporte Modelo mutuamente preferente.

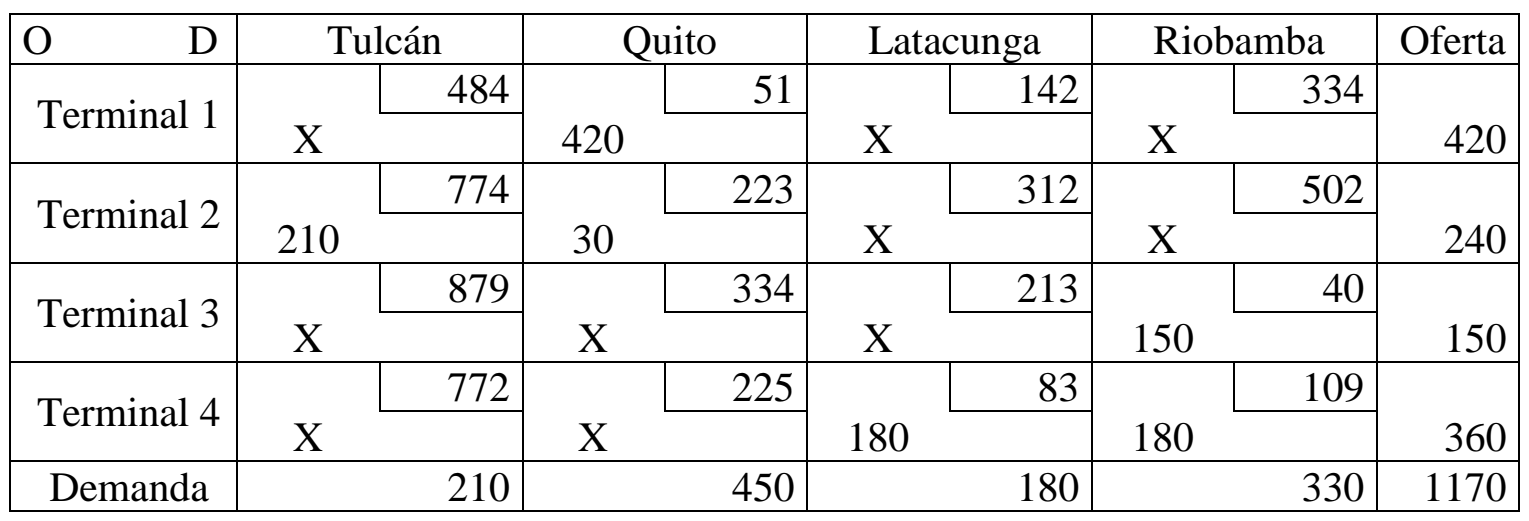

Fuente: Elaboración propia 


$$
\begin{gathered}
C T=420(51)+210(774)+30(223)+150(40)+180(83)+180(109) \\
C T=231.019,00
\end{gathered}
$$

\section{Análisis método Vogel}

En la Tabla 6, tenemos los resultados del método de transporte Vogel, en la que se observa una distribución similar al método del costo menor, en este caso a Tulcán se deberán enviar 210 tanqueros desde el terminal 1, a Quito se enviarán 210 tanqueros del terminal 1 y 240 tanqueros desde terminal 2, a Latacunga se deben enviar 180 tanqueros desde el terminal 4, y a Riobamba, se enviarán 150 tanqueros desde terminal 3, y 180 tanqueros desde el terminal 4. La función objetivo en este caso genera un costo de \$206354,00 dólares.

Tabla 6. Matriz de distribución de transporte Modelo Voguel.

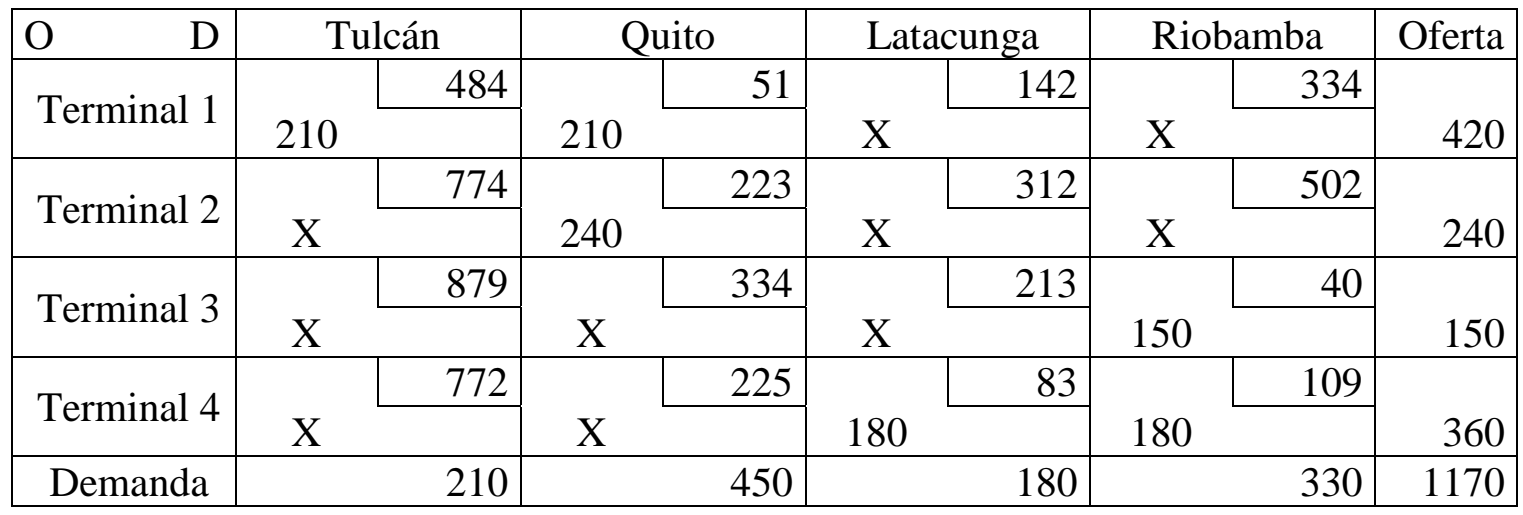

Fuente: Elaboración propia

$$
\begin{gathered}
C T=210(484)+210(51)+240(223)+150(40)+180(83)+180(109) \\
C T=206.354,00
\end{gathered}
$$

\section{Análisis método Russel}

La Tabla 7, muestra los resultados del método de transporte Russel, los cuales son similares al método Vogel, y al método del costo menor, por lo que la red planificada es la misma y genera un costo de $\$ 206354,00$ dólares. 
Tabla 7. Matriz de distribución de transporte Modelo Russel.

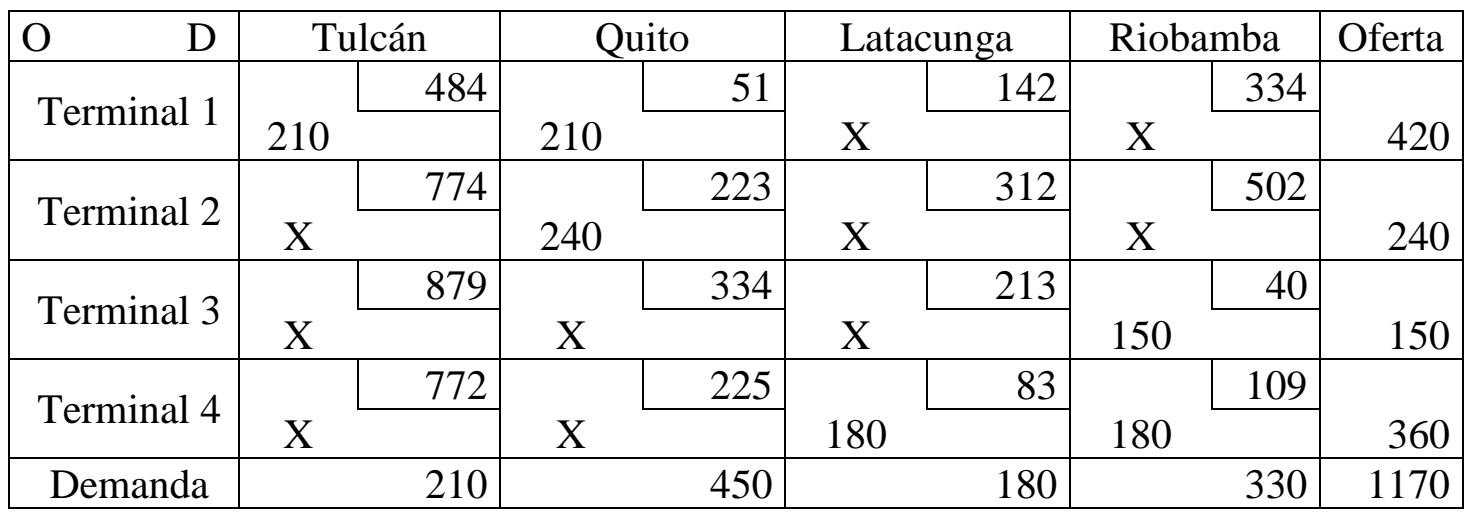

Fuente: Elaboración propia

$$
\begin{gathered}
C T=210(484)+210(51)+240(223)+150(40)+180(83)+180(109) \\
C T=206.354,00
\end{gathered}
$$

Con los datos obtenidos por los cinco métodos estudiados, y considerando las restricciones de cada uno de los orígenes y destinos, se puede concluir que el modelo matemático de transporte optimo para la empresa Comercializadora de combustibles, basado en programación lineal es el que presenta la estructura que minimiza los costos, en este caso la estructura que generan los métodos del costo menor, Vogel y Russel que forman la misma estructura, y cuya función objetivo en este caso genera un costo de $\$ 206354,00$ dólares.

\section{Conclusiones.}

- Para obtener los resultados esperados utilizando cualquiera de los métodos descritos lo más importante es realizar una adecuada descripción del problema y una identificación completa de las restricciones asociadas.

- Los modelos de transporte utilizados en el sector de hidrocarburos no son diferentes a los utilizados en otras industrias, se tratan de modelos de múltiples fuentes con múltiples destinos para Ecuador el tema se simplifica debido a la falta de infraestructura de transporte multimodal del país.

- El problema de la eficiencia de transporte no tiene una única solución los modelos analizados provee soluciones desde diferentes temas y Ciencias algunos están basados en la regresión lineal otros en fundamentos matemáticos como otros en ponderaciones y hasta en métodos empíricos esto para dar diferentes opciones de solución y poder elegir la óptima o la qué más genere valor frente a las necesidades. 
- Se debe tener en cuenta que los resultados obtenidos de los métodos aplicables nos enseñan cual es la mejor manera de modelar la programación de transporte y sus costos asociados.

\section{Referencias bibliográficas.}

Adame, W., Fontanilla, C., \& Arango, M. (2011). MODELOS LOGÍSTICOS PARA LA OPTIMIZACIÓN DEL TRANSPORTE DE. Ciencia e Ingeniería Neogranadina, 25. Obtenido de http://www.scielo.org.co/pdf/cein/v21n1/v21n1a06.pdf

García, G. (2014). Modelos para la planeacion de transporte terrestre eficiente. Universidad Militar Nueva Granada, 25. Obtenido de https://repository.unimilitar.edu.co/bitstream/handle/10654/11836/MODELOS\%20 PARA\%20LA\%20PLANEACION\%20DE\%20TRANSPORTE\%20TERRESTRE\% 20EFICIENTE\%20PARA\%20EL\%20SECTOR\%20DE\%20HIDROCARBUROS.p df;jsessionid=301D9D10EA65EE54F3D66712C4D8AC8E?sequence=1

Izar, J. (2012). Investigación de Operaciones. México: Trillas.

López, J., Olguín, J., \& Camargo, C. (2008). Modelo matemático de transporte aplicado a una compañía dedicada a la manufactura y distribución de juguetes, usando programación lineal entera. Revista Ingeniería Industrial, 8. Obtenido de https://dialnet.unirioja.es/servlet/articulo?codigo=3997971

Martínez, I., Vértiz, G., López, J., Jiménez, G., \& Moncayo, L. (2014). Investigación de Operaciones. México: Grupo Editorial Patria. Obtenido de http://editorialpatria.com.mx/pdffiles/9786074386967.pdf

Medina, S., Raya, K., \& Contreras, M. (2007). Utilización del modelo de transporte para la asignación de trabajos a máquinas considerando prioridades. Revista Académica de la FI-UADY, 9. Obtenido de https://www.redalyc.org/html/467/46711206/

Puente, M. I., \& Gavilánez, Ó. D. (2018). Programación lineal para la toma de decisiones. Riobamba: ESPOCH, Dirección de publicaciones.

Ruiz Estrada, G. (2004). Desarrollo de un Sistema de liberación de fármacos basado en nanopartículas magnéticas recubiertas con Polietilénglicol para el tratamiento de diferentes enfermedades. Madrid: Universidad Autónoma de Madrid. Departamento de Física Aplicada.

Taha, H. (2012). Investigación de operaciones. México: Pearson Educación. 
Tartaj, P., Morales, M., González-Carreño, T., Veintemillas-Verdaguer, S., \& Serna, C. (2005). Advances in magnetic nanoparticles for biotechnology applications. Journal of Magnetism and Magnetic Materials, 290, 28-34.

Wulff-Pérez , M., Martín-Rodriguez, A., Gálvez-Ruiz, M., \& de Vicente, J. ( 2013 ). The effect of polymer surfactant on the rheological properties of nanoemulsions. Colloid and Polymer Science, 291, 709-716.

Zamora Mora, V., Soares, P., Echeverria, C., Hernández, R., \& Mijangos, C. (2015). Composite chitosan/Agarose ferrogels for potential applications in magnetic hyperethermia. Gels., 1, 69-80.

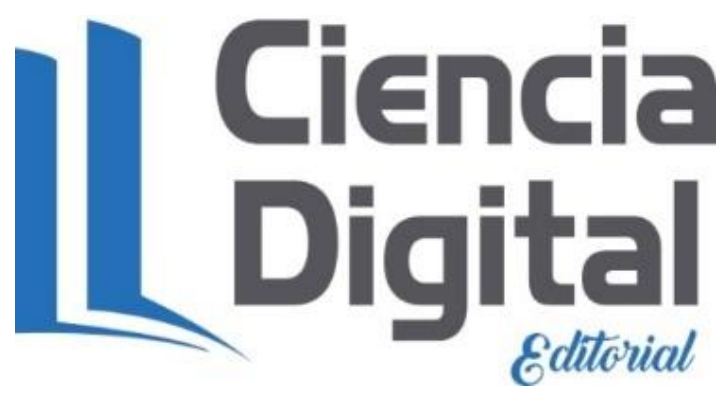


Para citar el artículo indexado.

Villamarín J., Aguilar G., Llamuca J. \& Villacrés W. (2019). Modelo matemático de transporte para una empresa comercializadora de combustibles, usando programación lineal Revista electrónica Ciencia Digital 3(2), 64-81. Recuperado desde: http://www.cienciadigital.org/revistascienciadigital/index.php/VisionarioDigital/article/vie $\underline{w / 394 / 878}$

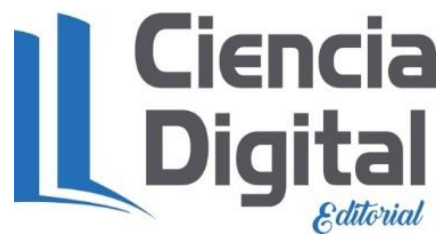

El artículo que se publica es de exclusiva responsabilidad de los autores y no necesariamente reflejan el pensamiento de la Revista Ciencia Digital.

El artículo queda en propiedad de la revista y, por tanto, su publicación parcial y/o total en otro medio tiene que ser autorizado por el director de la Revista Ciencia Digital.
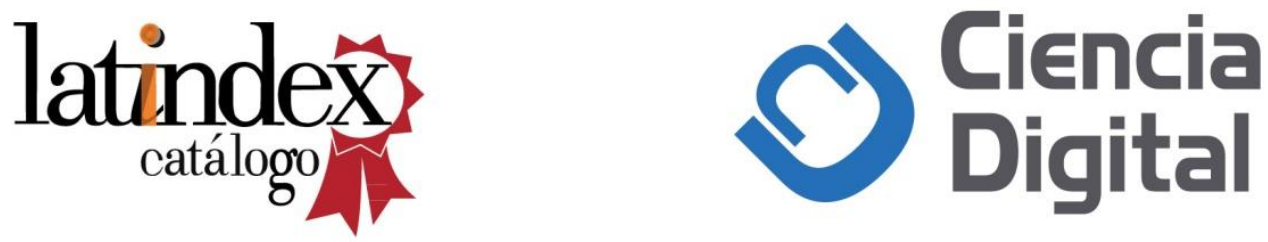